\title{
Volume-oriented routing and its modifications
}

\author{
Mateusz Żotkiewicz • Walid Ben-Ameur
}

Published online: 9 September 2011

(c) The Author(s) 2011. This article is published with open access at Springerlink.com

\begin{abstract}
Assuming that the traffic matrix belongs to a polytope, we present a new routing paradigm where each traffic demand is routed independently of the other demands: the volume-oriented routing. The routing of each demand is a combination of two extreme routing schemes depending on the current volume of the demand. This new routing paradigm is easy to implement in networks and quite efficient in terms of network cost. However, computing an optimal volume-oriented routing is generally difficult. Then, we introduce two modifications of the presented routing paradigm such that an optimal solution can be computed in polynomial time. Numerical experiments are also provided to compare volume-oriented routing with the best routing strategy in term of costs, i.e, dynamic routing.
\end{abstract}

Keywords Volume-oriented routing - Robust routing · Dynamic routing $\cdot$ Traffic demand polytope

\section{Introduction}

Due to the success of the Internet and the diversity of communication applications, it is becoming increasingly diffi-

This work was supported by the Polish Ministry of Science and Higher Education under Grants: N517 397334 and 280/N-DFG/2008/0.

M. Żotkiewicz $(\bowtie)$

Institute of Telecommunications, Warsaw University of Technology, Nowowiejska 15/19, 00-665 Warsaw, Poland

e-mail: mzotkiew@tele.pw.edu.pl

W. Ben-Ameur

Institut TELECOM, TELECOM SudParis, 9, rue Charles Fourier, 91011 Evry, France

e-mail: walid.benameur@it-sudparis.eu cult to forecast traffic patterns in modern telecommunication networks, as they have to deal with traffic generated by a variety of different applications utilized by a large number of users. Moreover, the customers' mobility makes a traffic demand matrix change in short periods of time. All those factors do not allow for considering only one static traffic demand matrix.

Some ideas to model the uncertainty in traffic demand matrices were proposed in the past. The first approach consists in building a traffic matrix based on the worst case for each traffic component. Routing is then computed based on this matrix. While this approach is simple, it can provide expensive solutions, because in fact it does not allow non-coincident traffic components to share resources.

The second approach is based on probabilistic modeling of traffic variations. After specifying such a model, one may look for routing that optimizes a probabilistic criterion: throughput expectation, average delays, blocking probability, etc. The solution obtained in this way is good on average, but can be very bad in some cases. Moreover, it requires knowledge of probability models, and they are usually difficult to obtain. This kind of approach is generally called stochastic programming, and was used for example in [18, 23], where a finite number of traffic scenarios with a known probability are considered.

Another variant of stochastic programming is chanceconstrained programming, where we look for a solution (a routing) satisfying the problem constraints with a certain probability. For example, given a probability distribution of the traffic matrix and a network with known capacities, we aim at determining a routing scheme such that the probability to block a link is lower than a small number $\varepsilon$. This approach is also generally difficult to apply since probabilities are not always easy to compute. The optimization problems 
that we get, when this approach is used, are very difficult to solve in exact way.

A different general approach called robust optimization (see [17]) takes into account a finite number of possible situations. In this case a solution that supports all situations is of interest. In the networking context, a routing scheme is to be determined such that each traffic matrix belonging to a given finite set of traffic matrices can be carried in the network.

Another robust model was proposed in [14] (and independently in [12]). It assumes that the outgoing traffic of each node is bounded (limitations on the incoming traffic can also be considered). Then the traffic matrix can be any matrix satisfying this kind of constraints. This uncertainty model is called the hose model. Several network design problems based on this model have received a considerable attention in the literature (see [10] and references therein).

A different model is considered in [8] where the authors assume that not all nodes can generate the maximum amount of traffic simultaneously. In fact this model, and the hose model presented above, are special cases of a more general polyhedral model considered in [3-5]. In this model all possible scenarios belong to a traffic demand polytope $D$, and our task is to provide a stable routing that is compatible with all traffic matrices of $D$ but not directly depending on the current traffic matrix. While stable routing is easy to implement, it can be expensive in terms of cost when compared to an optimal dynamic strategy where routing depends on the current traffic matrix. However, dynamic routing has two drawbacks: it is difficult to implement and is also difficult to compute as shown in [11].

To combine both robust static routing and dynamic routing, some improvements were proposed in [2] where the uncertainty set $D$ is partitioned into some subsets, and a robust routing is computed for each of them. This class of problems was considered in [6, 24]. Numerical experiments of [6, 24] show that partitioning strategies can sensitively reduce cost when compared to robust stable routing.

However, the drawback of those partitioning strategies is that they generally assume the centralization of routing. Moreover, the routing schemes corresponding to each subset can be very different, thus fluctuations may occur while switching from one routing to another. Therefore, it is worth considering something that will make the routing changes less abrupt, and does not require a global knowledge of the current traffic matrix.

In this paper we present a new routing paradigm, called Volume-Oriented Routing (and its polynomially solvable modifications: Simplified Volume-Oriented Routing and General Volume-Oriented Routing), that merges the simplicity of Robust Routing with the efficiency of Dynamic Routing. Moreover, it does not involve abrupt changes in network flows, and it is only based on local information. In order to support the paradigm, each node in a network has to be able to measure traffic originating in it. Moreover, a network has to be able to handle bifurcated flows.

Other robustness models and techniques to handle traffic uncertainty in networks can be found in $[9,13,18,19$, 21-23].

The paper is organized as follows. In Sect. 2 we present a notation and formulate Robust Routing, which is a starting point for our research. Robust Routing assumes that traffic matrices are always routed in the same way regardless of their locations in the traffic demand polytope. On the other hand, Dynamic Routing assumes that each traffic demand matrix can be routed independently of the others. This routing strategy is presented in Sect. 3. It is optimal in terms of cost, and can be seen as a lower bound for costs of any other strategy (including the strategies presented in this paper). In Sect. 4 traffic demand polytopes used in this paper are described. Then, in Sect. 5, Volume-Oriented Routing is presented. The computationally simpler cases, i.e., Simplified Volume-Oriented Routing and General VolumeOriented Routing, are covered in Sects. 6.1 and 6.2, respectively. The paper ends with numerical results in Sect. 7 and conclusions in Sect. 8.

\section{Robust Routing}

In this section a notation and the basic routing problem, denoted by Robust Routing, are presented.

\subsection{Notation}

We consider a directed graph $G=(\mathscr{V}, \mathscr{A})$, where $\mathscr{V}$ is the set of nodes and $\mathscr{A}$ is the set of arcs. The graph represents a backbone and the arcs depict unidirectional transmission links. For each arc $a \in \mathscr{A}$ an installed capacity $c_{a} \in \mathbb{R}_{+}$and a routing cost $w_{a} \in \mathbb{R}_{+}$for one unit of traffic are given.

Let $t=\left(t_{i j}\right)_{i, j \in \mathscr{V}}$ be a vector of $\mathbb{R}^{|\mathscr{V}|(|\mathscr{V}|-1)}$ that specifies values of traffic demands (or capacity requirements) between pairs of nodes of $\mathscr{V}$. This vector will be called a traffic matrix. A demand between nodes $u$ and $v$ is called "demand $u v$ ", and its value is denoted by $t_{u v}$. Note that the size of $t$ can be smaller when we assume that $t_{i j}=0$, for some $i, j \in \mathscr{V}$ and for all $t \in D$. The traffic matrix is supposed to be variable, and can be any point of a traffic demand polytope $D$. $D$ is generally defined by some linear constraints involving the variables $t_{i j}$, for $i, j \in \mathscr{V}$. However, it can be also defined by a set of traffic matrices (in this case $D$ is a convex hull of those matrices) or by an oracle.

To express routing problems as mathematical programs we introduce the following notation:

$\mathscr{P}(i, j)$ : finite set of acyclic paths of $G$ from $i$ to $j$ $(i, j \in \mathscr{V})$. 
$x_{p}^{i j}$ : proportion of volume of a demand $i j(i, j \in \mathscr{V})$ carried through a path $p \in \mathscr{P}(i, j)$. Note that $0 \leq$ $x_{p}^{i j} \leq 1$. For a current traffic matrix $t \in D$, the traffic carried through $p$ is then given by $t_{i j} \cdot x_{p}^{i j}$. To fulfill the stability property, the variable $x_{p}^{i j}$ does not directly depend on the current traffic matrix.

$x_{a}^{i j}$ : proportion of volume of a demand $i j(i, j \in \mathscr{V})$ flowing through an arc $a \in \mathscr{A}$.

$f_{a}$ : maximum amount of traffic carried on an arc $a \in \mathscr{A}$. It depends on the polytope $D$ and the routing pattern. The variable $f_{a}$ can also be considered as the minimum capacity that has to be reserved on an arc $a$ to satisfy all the constraints. A vector $f$ is called a reservation vector.

$w(D)$ : routing cost given by $\sum_{a \in \mathscr{A}} w_{a} f_{a}$.

\subsection{Problem formulation}

The problem of computing the minimum cost Robust Routing of an uncertainty domain $D$ can be formulated as in (1).

\section{Robust Routing}

$$
\begin{aligned}
& \text { Minimize: } w^{R R}(D)=\sum_{a \in \mathscr{A}} w_{a} f_{a} \\
& \sum_{p \in \mathscr{P}(i, j)} x_{p}^{i j} \geq 1 \quad \forall i, j \in \mathscr{V} \\
& \sum_{p \in \mathscr{P}(i, j), p \ni a} x_{p}^{i j} \leq x_{a}^{i j} \quad \forall i, j \in \mathscr{V}, \forall a \in \mathscr{A} \\
& \sum_{i, j \in \mathscr{V}} x_{a}^{i j} t_{i j} \leq f_{a} \quad \forall a \in \mathscr{A}, \forall t \in D \\
& f_{a} \leq c_{a} \quad \forall a \in \mathscr{A} \\
& x_{p}^{i j} \geq 0 \quad \forall p \in \mathscr{P}(i, j), \forall i, j \in \mathscr{V} \\
& x_{a}^{i j} \geq 0 \quad \forall a \in \mathscr{A}, \forall i, j \in \mathscr{V}
\end{aligned}
$$

The objective is to minimize the total routing cost. Inequalities (1a) express the fact that traffic demands between every pair of nodes may be split among many paths. Every variable $x_{a}^{i j}$ is defined by (1b). For a given traffic matrix $t$, a traffic on an arc $a$ is given by the left-hand side of (1c). Thus, the capacity $f_{a}$ that should be reserved on an arc $a$ must be higher than the traffic carried in all the situations. In other words, inequalities (1c) must be valid for each traffic matrix $t$ in the polytope $D$. Inequality (1d) indicates that $f_{a}$ is lower than the capacity of an $\operatorname{arc} a$. Note that all the variables have to be nonnegative.

Variables $x_{a}^{i j}$ can be eliminated from the formulation. However, according to our initial experiments eliminating them from the implementation does not improve running times of our algorithms. Therefore, we decided to keep them for the sake of clearness and simplicity.
Note that we can use other cost functions for this problem. For instance we can try to minimize the congestion. In such a situation the cost function has to be replaced by $w^{R R}(D)=z$, where $z$ is a variable denoting the congestion. We also have to replace (1d) by $f_{a} \leq z \cdot c_{a}$.

\subsection{Computational complexity}

Presented in this way, Robust Routing seems to be difficult. First, the number of paths of $\mathscr{P}(i, j)$ can be very high. Second, inequalities (1c) have to be satisfied for each traffic matrix in $D$, and $D$ is generally an infinite set. Fortunately, the problem was proved to be easily solvable using an algorithm based on constraint and path generation (see $[4,5])$.

Considering the current solution of the relaxation of Robust Routing (only a finite set of traffic matrices is taken into account instead of the full $D$ ), we only have to check whether there is an arc $a$ and a traffic matrix $t \in D$ such that (1c) is violated for them. This can be easily done by solving the following linear program:

$\max _{t \in D} \sum_{i \in \mathscr{V}} \sum_{j \in \mathscr{V} \backslash\{i\}} x_{a}^{i j} t_{i j}$.

If the maximum is larger than $f_{a}$ we add the violated inequality.

Routing paths can also be generated in an iterative way by solving a shortest path problem for each pair $(i, j)$, where $i, j \in \mathscr{V}$ and link weights are given by the values of appropriate dual variables of (1b). If there exists a path whose reduced cost is negative, it has to be added to an appropriate $\mathscr{P}(i, j)$.

Another way of solving Robust Routing, based on duality, was proposed in [1]. We should here consider the dual of the maximization problem above and write that the optimum must be always lower than $f_{a}$. A similar approach was also used in a previous work [7]. Instead of generating constraints, a compact formulation follows from duality but the number of variables of the problem increases significantly. Moreover, the approach assumes that a traffic demand polytope is given by a set of inequalities, while the approach presented in $[4,5]$ is more general and can also deal with polytopes described by their extreme points or by an oracle.

We should also notice that the problem difficulty does not change if we assume that either traffic demands or network links are not oriented. It is easy to solve the Robust Routing problem using the same kind of techniques even if either demands or links are undirected.

\section{Dynamic routing}

The problem consists in providing routing schemes (possibly different) for all traffic matrices from a traffic demand 
polytope. It is an optimal strategy in terms of costs, and will be used to evaluate the efficiency of the strategies presented in the following sections of the paper.

We need to introduce the following variables:

$x_{p}^{i j, t}:$ proportion of volume of a demand $i j(i, j \in \mathscr{V})$ for a traffic matrix $t$ carried through a path $p \in \mathscr{P}(i, j)$.

$x_{a}^{i j, t}:$ proportion of volume of a demand $i j(i, j \in \mathscr{V})$ for a traffic matrix $t$ on an arc $a \in \mathscr{A}$.

The core problem of this section is denoted as Dynamic Routing, and can be formulated as follows.

\section{Dynamic Routing}

$$
\begin{aligned}
& \text { Minimize: } \quad w^{D R}(D)=\sum_{a \in \mathscr{A}} w_{a} f_{a} \\
& \sum_{p \in \mathscr{P}(i, j)} x_{p}^{i j, t} \geq 1, \quad \forall i, j \in \mathscr{V}, \forall t \in D \\
& \sum_{p \in \mathscr{P}(i, j), p \ni a} x_{p}^{i j, t} \leq x_{a}^{i j, t}, \\
& \forall i, j \in \mathscr{V}, \forall a \in \mathscr{A}, \forall t \in D \\
& \sum_{i, j \in \mathscr{V}} x_{a}^{i j, t} t_{i j} \leq f_{a}, \quad \forall a \in \mathscr{A}, \forall t \in D \\
& f_{a} \leq c_{a}, \quad \forall a \in \mathscr{A} \quad \\
& x_{p}^{i j, t} \geq 0, \quad \forall p \in \mathscr{P}(i, j), \forall i, j \in \mathscr{V}, \forall t \in D \\
& x_{a}^{i j, t} \geq 0, \quad \forall a \in \mathscr{A}, \forall i, j \in \mathscr{V}, \forall t \in D^{\prime}
\end{aligned}
$$

Formulation (2) is similar to (1). However, it replaces each constraint of (1) involving variables $x_{p}^{i j}$ or $x_{a}^{i j}$ with an infinite (in general) number of constraints involving variables $x_{p}^{i j, t}$ and $x_{a}^{i j, t}$.

Although all traffic matrices $t \in D$ are present in the formulation, it is enough to only consider extreme points of $D$ in order to solve the problem. Having the optimal routings for all the extreme points it is possible to compute optimal routings for all $t \in D$ by calculating appropriate convex combinations of the optimal routings for the extreme points.

Remember that computing dynamic routing is generally a difficult problem. More precisely, answering the question whether a given traffic polytope can be dynamically routed through a known capacitated network is a co- $\mathscr{N} \mathscr{P}$ complete problem [11].

\section{Test cases}

In this section two different ways of describing a traffic demand polytope in practice are presented. We use both of them to evaluate our new routing strategies. The first is the hose model presented in [14]. The second is based on a model presented by Bertsimas and Sim in [8].

\subsection{Hose model}

In [14] a hose model was presented. It assumes that the outgoing traffic of each node is limited, i.e., $\sum_{j \in \mathscr{V}} t_{i j} \leq A_{i}$, for each $i \in \mathscr{V}$, where $A_{i}$ is an upper bound for outgoing traffic from a node $i$. Moreover, limitations on the incoming traffic can also be considered. In such a case, $\sum_{i \in \mathscr{V}} t_{i j} \leq B_{j}$, for each $j \in \mathscr{V}$, where $B_{j}$ is an upper bound for incoming traffic to a node $j$. The traffic matrix then can be any matrix satisfying this kind of constraints. A polytope satisfying those constraints is called a hose model polytope, and is formally described as follows.

\section{Hose model polytope}

$$
\begin{aligned}
& \sum_{j \in \mathscr{V}} t_{i j} \leq A_{i} \quad \forall i \in \mathscr{V} \\
& \sum_{i \in \mathscr{V}} t_{i j} \leq B_{j} \quad \forall j \in \mathscr{V}
\end{aligned}
$$

$t_{i j} \geq 0 \quad \forall i, j \in \mathscr{V}$

In addition it is possible to assume that both the maximum and minimum traffic between each pair of nodes are also constrained, i.e., $t_{i j}^{\min } \leq t_{i j} \leq t_{i j}^{\max }$. The obtained polytope is called a general hose model polytope, and is formally described as follows.

\section{General hose model polytope}

$\sum_{j \in \mathscr{V}} t_{i j} \leq A_{i} \quad \forall i \in \mathscr{V}, \forall t \in D$

$\sum_{i \in \mathscr{V}} t_{i j} \leq B_{j} \quad \forall j \in \mathscr{V}, \forall t \in D$

$t_{i j} \geq t_{i j}^{\min } \quad \forall i, j \in \mathscr{V}, \forall t \in D$

$t_{i j} \leq t_{i j}^{\max } \quad \forall i, j \in \mathscr{V}, \forall t \in D$

Note that not all nodes in a network have to generate traffic. Assume that some nodes are transit nodes, and are neither source nor sink nodes of any demand. They are called then non-active nodes, while the rest are called active nodes.

\subsection{B-S model}

In [8] Bertsimas and Sim presented a traffic demand model in which each demand can assume only either its minimum value $t_{i j}^{\min }$ or its maximum value $t_{i j}^{\max }$, and the number of demands that assume their maximum values cannot be greater than $k$. The model can be modified in a way that all possible traffic demand matrices considered in the original model are 
treated as extreme points of $D$ in a new model. A polytope satisfying those constraints is called $B$-S model polytope in this paper, and is formally described as follows.

\section{B-S model polytope}

$\sum_{i, j \in \mathscr{V}: t_{i j}^{\max }-t_{i j}^{\min } \neq 0} \frac{t_{i j}-t_{i j}^{\min }}{t_{i j}^{\text {max }}-t_{i j}^{\min }} \leq k \quad \forall t \in D$

$t_{i j} \geq t_{i j}^{\min } \quad \forall i, j \in \mathscr{V}, \forall t \in D$

$t_{i j} \leq t_{i j}^{\max } \quad \forall i, j \in \mathscr{V}, \forall t \in D$

Notice that, like in the hose model case, the number of extreme points of this traffic demand polytope is exponential. Moreover, also in this case we can talk about active and non-active nodes. For the sake of simplicity, assume that if a node is active, then it generates traffic to all other active nodes.

\section{Volume-Oriented Routing}

Volume-Oriented Routing is an extension of Robust Routing in which demands can be routed differently depending on their actual volumes. In this case the solution consists of two different routings for each demand and a set of thresholds. If a volume of a demand is smaller than a corresponding threshold the whole demand is routed using the first routing. If the volume is greater than the threshold a part of the demand equal to the threshold is sent using the first routing, while the rest of the demand uses the second routing.

\subsection{Notation}

In order to formulate the problem, the notation of Sect. 2.1 has to be extended by the following variables.

$h_{i j}$ : threshold for a demand $i j$.

$t_{i j}^{\prime}$ : volume of a demand $i j$ corresponding to a traffic demand matrix $t$ that has to be routed using the first routing scheme.

$t_{i j}^{\prime \prime}$ : volume of a demand $i j$ corresponding to a traffic demand matrix $t$ that has to be routed using the second routing scheme.

$\overline{x_{p}^{i j}}$ : routing scheme used by a demand $i j$ to route the volume exceeding $h_{i j}$ (second routing scheme).

$\overline{x_{a}^{i j}}$ : proportion of volume of a demand $i j$ exceeding $h_{i j}$ and flowing through an arc $a$.

\subsection{Problem formulation}

The problem of computing the minimum cost VolumeOriented Routing of an uncertainty domain $D$ can be formulated as in (6).
Volume-Oriented Routing

Minimize: $w^{V O}(D)=\sum_{a \in \mathscr{A}} w_{a} f_{a}$

$\sum_{p \in \mathscr{P}(i, j)} x_{p}^{i j} \geq 1 \quad \forall i, j \in \mathscr{V}$

$\sum_{p \in \mathscr{P}(i, j)} \overline{x_{p}^{i j}} \geq 1 \quad \forall i, j \in \mathscr{V}$

$\sum_{p \in \mathscr{P}(i, j), p \ni a} x_{p}^{i j} \leq x_{a}^{i j} \quad \forall i, j \in \mathscr{V}, \forall a \in \mathscr{A}$

$\sum_{p \in \mathscr{P}(i, j), p \ni a} \overline{x_{p}^{i j}} \leq \overline{x_{a}^{i j}} \quad \forall i, j \in \mathscr{V}, \forall a \in \mathscr{A}$

$\min \left(t_{i j}, h_{i j}\right)=t_{i j}^{\prime} \quad \forall i, j \in \mathscr{V}, \forall t \in D$

$\max \left(t_{i j}-h_{i j}, 0\right)=t_{i j}^{\prime \prime} \quad \forall i, j \in \mathscr{V}, \forall t \in D$

$\sum_{i, j \in \mathscr{V}}\left(x_{a}^{i j} t_{i j}^{\prime}+\overline{x_{a}^{i j}} t_{i j}^{\prime \prime}\right) \leq f_{a} \quad \forall a \in \mathscr{A}, \forall t \in D$

$f_{a} \leq c_{a} \quad \forall a \in \mathscr{A}$

$h_{i j} \geq 0 \quad \forall i, j \in \mathscr{V}$

$x_{p}^{i j}, \overline{x_{p}^{i j}} \geq 0 \quad \forall p \in \mathscr{P}(i, j), \forall i, j \in \mathscr{V}$

$x_{a}^{i j}, \overline{x_{a}^{i j}} \geq 0 \quad \forall a \in \mathscr{A}, \forall i, j \in \mathscr{V}$

In Problem (6) two different routings are considered for each demand (one used for the volume below the threshold and one used for the volume above the thresholds). Therefore, inequalities (1a) and (1b) have to be doubled into (6a), (6b) and (6c), (6d), respectively. The overall routing is expressed using (6e)-(6g). Equation (6e) (resp. (6f)) defines the volume that has to be routed using the first (resp. second) routing scheme (variables $x_{p}^{i j}$ (resp. $\overline{x_{p}^{i j}}$ ) and $x_{a}^{i j}$ (resp. $\left.\overline{x_{a}^{i j}}\right)$ ), while $(6 \mathrm{~g})$ defines the traffic sent on each link. Note that, Problems (6) and (1) are equivalent when either $h_{i j}=0$ or $h_{i j} \geq t_{i j}^{\max }$ (the maximum value of $t_{i j}$ ), for all $i, j \in \mathscr{V}$.

\subsection{Computational complexity}

Unfortunately a decision version of the problem is co- $\mathscr{N} \mathscr{P}$ complete, so we are not able to verify in polynomial time if a given solution is feasible. We will prove it in this section by reducing Subset Sum to the problem of verifying if a given solution is feasible.

Assume that a collection of numbers is given. Subset Sum consists in answering the question: is there a subset of those numbers whose sum is equal to a given limit. Subset Sum is $\mathscr{N} \mathscr{P}$-complete and can be considered as a special case of the Knapsack problem [16]. 


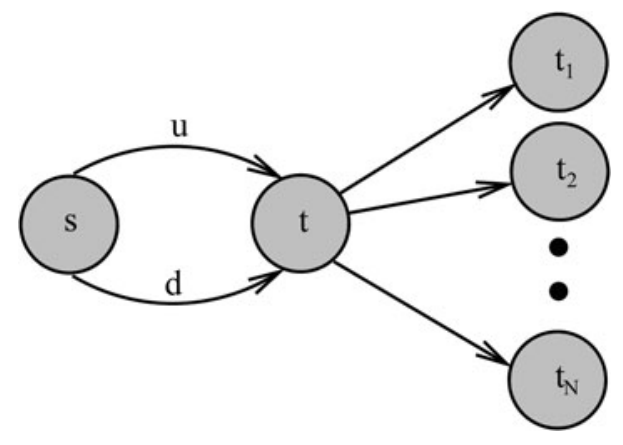

Fig. 1 Graph proving that a decision version of Volume-Oriented Routing is co- $\mathscr{N} \mathscr{P}$-complete

Consider a collection of integer numbers $\mathscr{L}=\left\{l_{1}, l_{2}, \ldots\right.$, $\left.l_{N}\right\}$ and an integer limit $L \geq 1$. The instance of Subset Sum is denoted by $S_{\mathscr{L}}$.

An instance $V_{\mathscr{L}}$ of Volume-Oriented Routing corresponding to $S \mathscr{L}$ is modeled by means of a graph presented in Fig. 1. Capacities of all links but one are unlimited. Only the capacity of a link $u$ is limited, and is equal to $\varepsilon \cdot(L-0.5)$, where $\varepsilon$ is a sufficiently small number, i.e., $0<\varepsilon<0.5$. Routing costs are set to 0 (in fact those values are irrelevant, because the feasibility of a solution is being verified, and not its actual cost). The traffic demand polytope $D$ describes possible volumes of $N$ different demands $s t_{i}$, where $i=1,2, \ldots, N$, each corresponding to one number from $\mathscr{L}$. The inequalities describing $D$ are as follows:

$t_{s t_{i}} \leq l_{i} \quad \forall i \in\{1,2, \ldots, N\}$

$\sum_{i=1}^{N} t_{s t_{i}} \leq L$

We want to know whether the following solution is feasible. The thresholds $h_{s t_{i}}$, for each demand $s t_{i}$, where $i=$ $1,2, \ldots, N$, are set to $l_{i} \cdot(1-\varepsilon)$. The first routing scheme (the one used for the volume below the threshold) for each demand uses link $d$, while the second routing scheme for each demand uses link $u$.

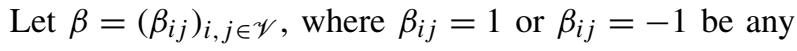
vector. Define $D_{\beta}$ as follows: $D_{\beta}=D \cap\left\{t, \forall_{i, j \in \mathscr{V}} \beta_{i j}\left(t_{i j}-\right.\right.$ $\left.\left.h_{i j}\right) \leq 0\right\}$. It is clear that $D=\bigcup_{\beta \in\{-\mathbf{1}, \mathbf{1}\}^{|\mathscr{Y}||\mathscr{V}|-\mathbf{1})}} D_{\beta}$. The solution considered above is not feasible if and only if there exists at least one $\beta \in\{-1,1\}^{|\mathscr{V}|(|\mathscr{V}|-1)}$ and a matrix $t \in D_{\beta}$ for which the routing is not feasible. Given any $\beta \in\{-1,1\}^{|\mathscr{V}|(|\mathscr{V}|-1)}$, the function $\left.\max \left(t_{i j}-h_{i j}, 0\right)\right)$ can be replaced by $t_{i j}-h_{i j}$ if $\beta_{i j}=-1$, or by 0 if $\beta_{i j}=1$, while function $\min \left(t_{i j}, h_{i j}\right)$ is equal to $t_{i j}$ when $\beta_{i j}=1$, and to $h_{i j}$ in the other case. In other words, constraint (6g) is linear in $t$ and has the same form for all matrices of $D_{\beta}$. Consequently, the existence of a matrix $t \in D_{\beta}$ for which the solution above is not feasible is equivalent to the existence of an extreme point of $D_{\beta}$ for which the same holds. To check feasibility, it is then possible to consider only extreme points of the family of polytopes $D_{\beta}$, where $\beta \in\{-1,1\}^{|\mathscr{V}|(|\mathscr{V}|-1)}$. This observation will be useful since the extreme points of $D_{\beta}$ have a very simple structure.

Lemma 1 The given solution to $V_{\mathscr{L}}$ is feasible if and only if the answer to $S_{\mathscr{L}}$ is $N O$.

Proof Suppose that the answer to $S_{\mathscr{L}}$ is YES. Then the traffic demand polytope $D$ contains at least one extreme point such that $\forall_{i=1,2, \ldots, N} t_{s t_{i}} \in\left\{0, l_{i}\right\}$, and $\sum_{i=1}^{N} t_{s t_{i}}=L$. Such an extreme point requires $\varepsilon \cdot L$ of capacity at the link $u$, which is $0.5 \cdot \varepsilon$ more than the available capacity. Therefore, the given solution to $V_{\mathscr{L}}$ is not feasible.

Now assume that the solution is unfeasible, and let us prove that the answer to $S_{\mathscr{L}}$ is YES. There exists a traffic demand matrix $t \in D$ and a routing corresponding to this matrix such that together they require more than $\varepsilon \cdot(L-0.5)$ of capacity on the link $u$. According to the observation above, the feasibility has to be only checked for the extreme points of the family of polytopes $D_{\beta}$. Each $D_{\beta}$ is described by sets of constraints but only one of those constraints contains more than one variable. Therefore, for each extreme point of any $D_{\beta}$, an equation $t_{s t_{i}}=\left\{l_{i}, l_{i} \cdot(1-\varepsilon), 0\right\}$ can be not satisfied only for one $t_{s t_{i}}$, where $i=1,2, \ldots, N$. Note that $l_{i} \cdot(1-\varepsilon)$ is the value of the threshold for a demand $s t_{i}$.

Assume that $t_{s t_{1}}=l_{1} \cdot(1-\varepsilon)$. Note that in this case $t_{s t_{1}}=$ $h_{s t_{1}}$ so the whole $t_{s t_{1}}$ is routed using a link $d$. The rest of the available demands' volume, i.e., at most $L-l_{1} \cdot(1-\varepsilon)$ [as a result of (7b)], has to be routed in such a way that it will use more than $\varepsilon \cdot(L-0.5)$ of the capacity of the link $u$. But $\max _{t_{s t_{i}} \in\left[0, l_{i}\right]} \frac{t_{s t_{i}}-h_{s t_{i}}}{t_{s t_{i}}}=\varepsilon$, for $i=1,2, \ldots, N$. Therefore, the remaining volume cannot use more than $\varepsilon \cdot\left(L-l_{1}+\varepsilon \cdot l_{1}\right)$ of the capacity of the link $u$, and it is less than $\varepsilon \cdot(L-$ $0.5)$, because we assumed that $l_{1}$ is an integral number and $\varepsilon<0.5$.

Knowing that $\forall_{i=1,2, \ldots, N} t_{s t_{i}} \neq l_{i} \cdot(1-\varepsilon)$, we can conclude that for any extreme point of any $D_{\beta}$ for which the routing solution is not feasible, an equation $t_{s t_{i}}=\left\{l_{i}, 0\right\}$ has to be satisfied for at least all but one $t_{s t_{i}}$. Assume that the equation is not satisfied for $i=N$. Then constraint (7b) is necessarily saturated. In other terms, we have $M+t_{s t_{N}}=L$, where $M$ is the sum of volumes of the other demands. Notice that this implies $t_{s t_{N}}$ is integer. The demands other than $t_{s t_{N}}$ consume at most $\varepsilon \cdot\left(L-t_{s t_{N}}\right)$ of the capacity of the link $u$, so at least $\varepsilon \cdot\left(t_{s t_{N}}-0.5\right)$ has to be consumed by the demand $s t_{N}$. Using (7a) and knowing that $h_{s t_{N}}=l_{N} \cdot(1-\varepsilon)$ we can write that $\left(t_{s t_{N}}-h_{s t_{N}}\right) \geq \varepsilon \cdot\left(t_{s t_{N}}-0.5\right)$. In other words, we have $l_{N} \geq t_{s t_{N}} \geq l_{N}-\frac{0.5 \cdot \varepsilon}{1-\varepsilon}$. Knowing that $t_{s t_{N}}$ is integer and $\frac{0.5 \cdot \varepsilon}{1-\varepsilon}<1$ we conclude that $t_{s t_{N}}=l_{N}$. It means that the answer to $S_{\mathscr{L}}$ is YES.

To finish the proof we have to consider the case where $t_{s t_{i}}=\left\{l_{i}, 0\right\}$ is satisfied for each traffic component. If the 
constraint (7b) is saturated, then the answer to $S_{\mathscr{L}}$ is still YES. Suppose that $\sum_{i} t_{s t_{i}}<L$. Since all $t_{s t_{i}}$ are integer in this case (either equal to 0 or $l_{i}$ ), the previous inequality becomes $\sum_{i} t_{s t_{i}} \leq L-1$. Then the traffic sent on link $u$ is less than $\varepsilon \cdot(L-1)$. However, the routing solution is not feasible implying that this traffic is more than $\varepsilon \cdot(L-0.5)$. We clearly get a contradiction. Said another way, The answer to $S_{\mathscr{L}}$ is always YES whenever the proposed solution to $V_{\mathscr{L}}$ is not feasible.

Proposition 1 The decision version of Volume-Oriented Routing is co- $\mathscr{N} \mathscr{P}$-complete.

Proof The feasibility problem is in co- $\mathscr{N} \mathscr{P}$ since a certificate of a negative response is given by any matrix that cannot be routed according to the proposed thresholds and routing schemes. It is easy to check whether a given matrix can be routed according to some given routing schemes. The reduction proposed in Lemma 1 ends the proof.

As Volume-Oriented Routing is difficult, we will consider some of its interesting special cases that lead to easily solvable problems. They will be discussed in the following sections. Note that, according to Sect. 7, the special case presented in Sect. 6.1 (and its modification presented in Sect. 6.2) is very efficient.

\section{Modifications}

In this section we present two novel strategies. The first is called Simplified Volume-Oriented Routing and is a simplification of Volume-Oriented Routing. The second strategy is called General Volume-Oriented Routing. It can be seen as a generalization of Simplified Volume-Oriented Routing. However, it is not a simplification of Volume-Oriented Routing anymore. Thus, Simplified Volume-Oriented Routing and General Volume-Oriented Routing cannot be considered as simplifications or generalizations of each other.

\subsection{Simplified Volume-Oriented Routing}

The strategy considered in this section is a special case of Volume-Oriented Routing. In the latter case, positions of the thresholds are subject to optimization, while in the former case those positions are set to the minimum values of the corresponding demands' volumes. The change makes the problem polynomial.

Let us define $t_{i j}^{\min }$ as $\min _{t \in D} t_{i j}$, and $t_{i j}^{\max }$ as $\max _{t \in D} t_{i j}$. The formulation of Simplified Volume-Oriented Routing modifies (6) by simplifying definitions of $t_{i j}^{\prime}$ and $t_{i j}^{\prime \prime}$, i.e., it replaces (6e) and (6f) with new constraints. The formulation is as in (8).

\section{Simplified Volume-Oriented Routing}

Minimize: $\quad w^{S V O}(D)=\sum_{a \in \mathscr{A}} w_{a} f_{a}$

$\sum_{p \in \mathscr{P}(i, j)} x_{p}^{i j} \geq 1 \quad \forall i, j \in \mathscr{V}$

$\sum_{p \in \mathscr{P}(i, j)} \overline{x_{p}^{i j}} \geq 1 \quad \forall i, j \in \mathscr{V}$

$\sum_{p \in \mathscr{P}(i, j), p \ni a} x_{p}^{i j} \leq x_{a}^{i j} \quad \forall i, j \in \mathscr{V}, \forall a \in \mathscr{A}$

$\sum_{p \in \mathscr{P}(i, j), p \ni a} \overline{x_{p}^{i j}} \leq \overline{x_{a}^{i j}} \quad \forall i, j \in \mathscr{V}, \forall a \in \mathscr{A}$

$t_{i j}^{m i n}=t_{i j}^{\prime} \quad \forall i, j \in \mathscr{V}, \forall t \in D$

$t_{i j}-t_{i j}^{\text {min }}=t_{i j}^{\prime \prime} \quad \forall i, j \in \mathscr{V}, \forall t \in D$

$\sum_{i, j \in \mathscr{V}}\left(x_{a}^{i j} t_{i j}^{\prime}+\overline{x_{a}^{i j}} t_{i j}^{\prime \prime}\right) \leq f_{a} \quad \forall a \in \mathscr{A}, \forall t \in D$

$f_{a} \leq c_{a} \quad \forall a \in \mathscr{A}$

$x_{p}^{i j}, \overline{x_{p}^{i j}} \geq 0 \quad \forall p \in \mathscr{P}(i, j), \forall i, j \in \mathscr{V}$

$x_{a}^{i j}, \overline{x_{a}^{i j}} \geq 0 \quad \forall a \in \mathscr{A}, \forall i, j \in \mathscr{V}$

Moreover, as threshold variables $h_{i j}$ are not used, inequalities (6i) are obsolete for Simplified Volume-Oriented Routing.

Notice that, if $t_{i j}^{\text {min }}=0$ for all $i, j \in \mathscr{V}$ then Simplified Volume-Oriented Routing is equivalent to Robust Routing. Then it is better to consider $t_{i j}^{\text {min }}$ over non-dominated traffic demand matrices from $D$, and not over the whole polytope $D$.

The problem is polynomial, and can be easily solved using techniques presented in Sect. 2.3, i.e., using an algorithm based on constraint generation (see $[4,5]$ ) and path generation in an iterative way.

\subsection{General Volume-Oriented Routing}

The strategy considered in this section can be seen as an extension of Simplified Volume-Oriented Routing, with more general routing schemes.

The formulation of General Volume-Oriented Routing is quite similar to the formulation of its predecessor, i.e., Simplified Volume-Oriented Routing. The only differences are in definitions of $t_{i j}^{\prime}$ and $t_{i j}^{\prime \prime}$. The formulation of General Volume-Oriented Routing is as in (9).

\section{General Volume-Oriented Routing}

Minimize: $\quad w^{G V O}(D)=\sum_{a \in \mathscr{A}} w_{a} f_{a}$ 
$\sum_{p \in \mathscr{P}(i, j)} x_{p}^{i j} \geq 1 \quad \forall i, j \in \mathscr{V}$

$\sum_{p \in \mathscr{P}(i, j)} \overline{x_{p}^{i j}} \geq 1 \quad \forall i, j \in \mathscr{V}$

$\sum_{p \in \mathscr{P}(i, j), p \ni a} x_{p}^{i j} \leq x_{a}^{i j} \quad \forall i, j \in \mathscr{V}, \forall a \in \mathscr{A}$

$\sum_{p \in \mathscr{P}(i, j), p \ni a} \overline{x_{p}^{i j}} \leq \overline{x_{a}^{i j}} \quad \forall i, j \in \mathscr{V}, \forall a \in \mathscr{A}$

$\alpha_{i j} t_{i j}+\beta_{i j}=t_{i j}^{\prime} \quad \forall i, j \in \mathscr{V}, \forall t \in D$

$\left(1-\alpha_{i j}\right) t_{i j}-\beta_{i j}=t_{i j}^{\prime \prime} \quad \forall i, j \in \mathscr{V}, \forall t \in D$

$\sum_{i, j \in \mathscr{V}}\left(x_{a}^{i j} t_{i j}^{\prime}+\overline{x_{a}^{i j}} t_{i j}^{\prime \prime}\right) \leq f_{a} \quad \forall a \in \mathscr{A}, \forall t \in D$

$f_{a} \leq c_{a} \quad \forall a \in \mathscr{A}$

$x_{p}^{i j}, \overline{x_{p}^{i j}} \geq 0 \quad \forall p \in \mathscr{P}(i, j), \forall i, j \in \mathscr{V}$

$x_{a}^{i j}, \overline{x_{a}^{i j}} \geq 0 \quad \forall a \in \mathscr{A}, \forall i, j \in \mathscr{V}$

Where vectors $\alpha$ and $\beta$ are given, and satisfy the following set of constraints.

$\alpha_{i j} t_{i j}^{\text {min }}+\beta_{i j} \geq 0 \quad \forall i, j \in \mathscr{V}$

$\left(1-\alpha_{i j}\right) t_{i j}^{\min }-\beta_{i j} \geq 0 \quad \forall i, j \in \mathscr{V}$

$\alpha_{i j} t_{i j}^{\max }+\beta_{i j} \geq 0 \quad \forall i, j \in \mathscr{V}$

$\left(1-\alpha_{i j}\right) t_{i j}^{\max }-\beta_{i j} \geq 0 \quad \forall i, j \in \mathscr{V}$

It is obvious that Simplified Volume-Oriented Routing is a special case of General Volume-Oriented Routing, where $\alpha_{i j}=0$ and $\beta_{i j}=t_{i j}^{\min }$, for each $i, j \in \mathscr{V}$. An interesting observation is that for each $D$ there exists a simple way to obtain an optimal pair of vectors $\alpha$ and $\beta$.

Proposition 2 For a given $D$, vectors $\alpha$ and $\beta$ defined in (11) yield an optimal solution, i.e., there are no cheaper solutions to General Volume-Oriented Routing for any other $\alpha$ and $\beta$.

$\alpha_{i j}=\frac{-t_{i j}^{\min }}{t_{i j}^{\max }-t_{i j}^{\min }} \quad \forall i, j \in \mathscr{V}$

$\beta_{i j}=\frac{t_{i j}^{\max } t_{i j}^{\min }}{t_{i j}^{\max }-t_{i j}^{\min }} \quad \forall i, j \in \mathscr{V}$

Proof Consider a capacity consumed by only one demand $i j$. The constraints defining the used capacity on a link $a$ for General Volume-Oriented Routing can be combined into:

$x_{a}^{i j}\left(\alpha_{i j} t_{i j}+\beta_{i j}\right)+\overline{x_{a}^{i j}}\left[\left(1-\alpha_{i j}\right) t_{i j}-\beta_{i j}\right]$

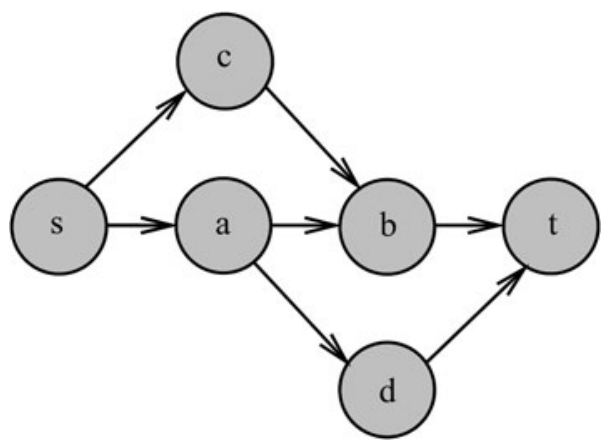

Fig. 2 Example proving that General Volume-Oriented Routing outperforms Simplified Volume-Oriented Routing when the hose and B-S models are of interest

and for $\alpha$ and $\beta$ defined as (11) into:

$z_{a}^{i j} t_{i j}^{\min } \frac{t_{i j}^{\max }-t_{i j}}{t_{i j}^{\max }-t_{i j}^{\min }}+\bar{z}_{a}^{i j} t_{i j}^{\max } \frac{t_{i j}-t_{i j}^{\min }}{t_{i j}^{\max }-t_{i j}^{\min }}$

For the sake of clearness, in the latter case, $z_{a}^{i j}$ was substituted for $x_{a}^{i j}$ and $\overline{z_{a}^{i j}}$ for $\overline{x_{a}^{i j}}$. Assume that the routing for General Volume-Oriented Routing (with $\alpha$ and $\beta$ denoted by $\alpha^{\prime}$ and $\beta^{\prime}$ ) is known and expressed using vectors $x$ and $\bar{x}$. In order to obtain the same results for all $t \in D$ and suggested $\alpha$ and $\beta$ take:

$z_{a}^{i j}=\left(\alpha_{i j}^{\prime}+\frac{\beta_{i j}^{\prime}}{t_{i j}^{\min }}\right) x_{a}^{i j}+\left(1-\alpha_{i j}^{\prime}-\frac{\beta_{i j}^{\prime}}{t_{i j}^{\min }}\right) \overline{x_{a}^{i j}}$

$\overline{z_{a}^{i j}}=\left(\alpha_{i j}^{\prime}+\frac{\beta_{i j}^{\prime}}{t_{i j}^{\text {max }}}\right) x_{a}^{i j}+\left(1-\alpha_{i j}^{\prime}-\frac{\beta_{i j}^{\prime}}{t_{i j}^{\text {max }}}\right) \overline{x_{a}^{i j}}$

It is an easy exercise to check that the obtained load on each link will be exactly equal to what is obtained when General Volume-Oriented Routing is considered. Moreover, the routing is feasible, because both $z_{a}^{i j}$ and $z_{a}^{i j}$ are convex combinations of $x_{a}^{i j}$ and $\overline{x_{a}^{i j}}$, for $a \in \mathscr{A}$ and $i, j \in \mathscr{V}$. Note that $0 \leq \alpha_{i j}^{\prime}+\frac{\beta_{i j}^{\prime}}{t_{i j}^{m i n}} \leq 1$, and $0 \leq \alpha_{i j}^{\prime}+\frac{\beta_{i j}^{\prime}}{t_{i j}^{\text {max }}} \leq 1$, for all $i, j \in \mathscr{V}$, because of (10).

Alike Simplified Volume-Oriented Routing, its modification, i.e., General Volume-Oriented Routing can be solved in polynomial time using the techniques presented in $[4,5]$.

Obviously, General Volume-Oriented Routing is able to produce routing schemes unavailable for Simplified VolumeOriented Routing, e.g., for $t_{i j}=t_{i j}^{\text {min }}$ the whole demand $i j$ is routed using one link, while for $t_{i j}=t_{i j}^{\max }$ the whole demand $i j$ is routed using another link. Thus, the two approaches are not equivalent. We give below an example showing that the approaches can be different even if either the B-S model or the hose model is assumed. 
Example 1 Consider a network presented in Fig. 2 with $c_{u}=1$, for all $u \in \mathscr{A}$. Consider three non-zero demands: $s t, s c$, and $d t$. The constraints of the B-S model are as follows: $t_{s t}^{\text {min }}=1, t_{s t}^{\text {max }}=2, t_{s c}^{\text {min }}=0, t_{s c}^{\text {max }}=1, t_{d t}^{\text {min }}=0$, and $t_{d t}^{\max }=1$. Assume $k=1$. In such a network, Simplified Volume-Oriented Routing has no solution, while the only feasible solution for General Volume-Oriented Routing uses a path $s-a-b-t$ for the first routing of the demand $s t$, and paths $s-c-b-t$ and $s-a-d-t$ for the second routing.

The same result can be obtained for a general hose model polytope specified as follows: four active nodes $\{s, t, c, d\}$, $A_{s}=2, B_{s}=0, A_{t}=0, B_{t}=2, A_{c}=0, B_{c}=1, A_{d}=1$, $B_{d}=0$, and $t_{d c}^{\max }=0$. For a classical hose model polytope the bound on $t_{d c}^{\max }$ has to be substituted by an additional link of capacity 1 joining $d$ and $s$.

\section{Numerical results}

We tested our strategies on an Intel $2.4 \mathrm{GHz} \mathrm{CPU}$ with 3.25 GB RAM, using a linear programming solver CPLEX 11.0 [15]. We built our example cases using real world networks available in SNDlib [20]. We used atlanta and france topologies. Both were tested using two different sets of active nodes $\mathscr{V}^{a c t}$, and five different traffic demand polytopes defined for each of the sets. The first polytope for each set satisfied restrictions of the hose model presented in Sect. 4.1. The sets of active nodes and the bounds for incoming and outgoing traffic were chosen at random. The minimum traffic between a pair of node $i$ and $j$, where $i, j \in \mathscr{V}^{a c t}$, was set to $\frac{\min \left(A_{i}, B_{j}\right)}{2 \cdot\left|\mathscr{V}^{a c t}\right|}$, and the maximum traffic was set to $\frac{\min \left(A_{i}, B_{j}\right)}{2}$, where $A_{i}$ and $B_{j}$ are defined as in Sect. 4.1. Another two traffic demand polytopes were convex hulls of a number ( 3 or 10) of extreme points of the first polytope. The last two polytopes were built using the B-S polytope model presented in Sect. 4.2. The bounds were set at random, while $k$ was set to $10 \%$ or $20 \%$ of its maximum value, i.e., 0.1 or 0.2 times $\left|\mathscr{V}^{a c t}\right| \cdot\left(\left|\mathscr{V}^{a c t}\right|-1\right)$, depending on the test case.

We used an evaluation strategy similar to the one presented in [6]. First, the polytopes were scaled in such a way that the congestion, using Robust Routing, obtained for the most loaded link was $110 \%$. Then, in order to handle the insolvability of our test cases (we build them in such a way that all networks are congested) additional uncapacitated arcs between all pairs of active nodes were introduced. Routing costs of these additional arcs were set in such a way that it was approximately twenty times more expensive to use them than paths consisting of arcs in the original network. We can consider those arcs as possibilities to rent capacity from other operators or as links set up using different, and more expensive, technologies, e.g., satellite links.
The result of our experiments are presented in Table 1. It consists of fourteen columns. The first five describe test cases, and are as follows:

topology: name of the topology in SNDLib.

$|\mathscr{V}|$ : number of nodes in the network.

$|\mathscr{A}|$ : number of undirected links in the network. Note that, additional expensive links are not included here.

dim. $D$ : dimension of the traffic demand polytope, i.e., $\left|\mathscr{V}^{a c t}\right| \cdot\left(\left|\mathscr{V}^{\text {act }}\right|-1\right)$.

variant: way a traffic demand polytope was created, hose-full hose model polytope, 3 pts. (10 pts.) convex hull of three (ten) random extreme points of a full hose model polytope, B-S $10 \%$ (resp. $20 \%$-B-S polytope model with $k=\left\lfloor K \cdot\left|\mathscr{V}^{a c t}\right|\right.$. $\left.\left(\left|\mathscr{V}^{a c t}\right|-1\right)\right\rfloor$, where $K=0.1$ (resp. 0.2).

The next nine columns contain costs, gains (computed by dividing the cost difference between Robust Routing and the two volume-oriented strategies of Sect. 6 by the cost difference between Robust Routing and the lower bound), and computing times of all optimization problems presented in the paper. The following problems are considered:

Robust Routing: strategy described in Sect. 2. It is the simplest routing strategy and an upper bound on costs of all other strategies.

Lower bound: lower bound on a cost of all strategies. For 3 pts. and 10 pts. we used Dynamic Routing. Note that computing optimal Dynamic Routing is extremely difficult as it requires a set of routing variables for each extreme point of a traffic demand polytope. Therefore, for hose, B-S $10 \%$, and B-S $20 \%$ we decided to compute it for a random set of 50 extreme points instead of taking into account the whole $D$.

SVO-Routing: strategy presented in Sect. 6.1.

GVO-Routing: strategy presented in Sect. 6.2, with the optimal values of vectors $\alpha$ and $\beta$.

It is clearly seen that both tested strategies are very efficient, and in most cases give at least $50 \%$ of the maximum possible gain, i.e., the gain given by Dynamic Routing. Moreover, in one case they are as efficient as the mentioned Dynamic Routing. Notice that the gains presented in this table are generally (for hose, B-S 10\%, and B-S 20\%) lower bounds of the real gain since we are only able to obtain a lower bound for the cost of Dynamic Routing.

It is worth to notice that there is no difference between Simplified Volume-Oriented Routing and General VolumeOriented Routing for the hose and B-S models polytopes. It may leave an impression that General Volume-Oriented Routing is useless as far as those models are concerned. However, remember that an example was given at the end of Sect. 6 showing that Simplified Volume-Oriented Routing and General Volume-Oriented Routing are generally not 
Table 1 Performance of the presented approaches

\begin{tabular}{|c|c|c|c|c|c|c|c|c|c|c|c|c|c|}
\hline \multicolumn{5}{|l|}{ network } & \multicolumn{2}{|c|}{ Robust Routing } & \multirow{2}{*}{$\begin{array}{l}\text { Lower bound } \\
\text { cost }\end{array}$} & \multicolumn{3}{|c|}{ SVO-Routing } & \multicolumn{3}{|c|}{ GVO-Routing } \\
\hline topology & $|\mathscr{V}|$ & $|\mathscr{A}|$ & $\operatorname{dim} . D$ & variant & cost & time $[\mathrm{s}]$ & & cost & gain[\%] & time $[\mathrm{s}]$ & cost & gain[\%] & time $[\mathrm{s}]$ \\
\hline \multirow[t]{10}{*}{ atlanta } & 15 & 44 & 12 & 3 pts. & 723.0 & 0.1 & 715.6 & 717.5 & 74.3 & 0.4 & 717.5 & 74.3 & 0.2 \\
\hline & & & & 10 pts. & 447.5 & 0.2 & 329.1 & 332.0 & 97.6 & 0.4 & 332.0 & 97.6 & 0.3 \\
\hline & & & & hose & 442.3 & 0.3 & 317.7 & 380.3 & $\geq 49.8$ & 0.5 & 380.3 & $\geq 49.8$ & 0.5 \\
\hline & & & & B-S $10 \%$ & 361.1 & 0.1 & 286.7 & 287.9 & $\geq 98.4$ & 0.2 & 287.9 & $\geq 98.4$ & 0.2 \\
\hline & & & & B-S $20 \%$ & 343.0 & 0.2 & 193.6 & 291.8 & $\geq 34.3$ & 0.3 & 291.8 & $\geq 34.3$ & 0.5 \\
\hline & & & 30 & 3 pts. & 410.7 & 0.6 & 389.6 & 395.2 & 73.5 & 0.7 & 393.5 & 81.5 & 0.9 \\
\hline & & & & 10 pts. & 448.8 & 0.7 & 400.6 & 420.5 & 58.7 & 1.6 & 420.4 & 58.9 & 2.5 \\
\hline & & & & hose & 443.5 & 3.1 & 265.7 & 402.1 & $\geq 23.3$ & 7.0 & 402.1 & $\geq 23.3$ & 9.9 \\
\hline & & & & B-S $10 \%$ & 625.7 & 1.0 & 264.5 & 350.6 & $\geq 76.2$ & 6.1 & 350.6 & $\geq 76.2$ & 10.5 \\
\hline & & & & B-S $20 \%$ & 506.1 & 1.4 & 315.3 & 448.0 & $\geq 30.5$ & 11.0 & 448.0 & $\geq 30.5$ & 25.9 \\
\hline \multirow[t]{10}{*}{ france } & 25 & 90 & 20 & 3 pts. & 332.4 & 0.6 & 317.8 & 317.8 & 100.0 & 0.7 & 317.8 & 100.0 & 0.8 \\
\hline & & & & 10 pts. & 353.6 & 0.8 & 320.3 & 324.1 & 88.6 & 0.8 & 324.1 & 88.6 & 1.6 \\
\hline & & & & hose & 357.0 & 1.6 & 321.4 & 328.9 & $\geq 78.9$ & 1.9 & 328.9 & $\geq 78.9$ & 3.0 \\
\hline & & & & B-S $10 \%$ & 394.5 & 1.4 & 263.9 & 371.5 & $\geq 17.6$ & 2.7 & 371.5 & $\geq 17.6$ & 4.3 \\
\hline & & & & B-S $20 \%$ & 483.6 & 1.7 & 296.9 & 477.9 & $\geq 3.1$ & 4.9 & 477.9 & $\geq 3.1$ & 8.8 \\
\hline & & & 56 & 3 pts. & 652.3 & 3.6 & 614.1 & 619.6 & 85.6 & 3.7 & 619.3 & 86.4 & 3.4 \\
\hline & & & & 10 pts. & 695.4 & 8.6 & 520.5 & 574.2 & 69.3 & 22.7 & 565.1 & 74.5 & 37.3 \\
\hline & & & & hose & 649.2 & 495.0 & 278.3 & 525.4 & $\geq 33.4$ & 1336.4 & 525.4 & $\geq 33.4$ & 3401.0 \\
\hline & & & & B-S $10 \%$ & 854.5 & 33.2 & 314.0 & 802.8 & $\geq 9.6$ & 177.4 & 802.8 & $\geq 9.6$ & 765.1 \\
\hline & & & & B-S $20 \%$ & 518.7 & 11.5 & 261.1 & 516.3 & $\geq 0.9$ & 605.7 & $\leq 516.3$ & $\geq 0.9$ & 7200.0 \\
\hline
\end{tabular}

equivalent when either the hose or the B-S models are considered.

Going back to the computational results, the obtained running times are satisfactory, and prove that the strategies can be successfully implemented in medium size networks. The 7200-second time limit was hit only once. However, we were still able to obtain a feasible solution to the problem (that is why the cost is presented using a sign $\leq$ ).

\section{Conclusion}

In the paper we presented a novel routing paradigmVolume-Oriented Routing that is supposed to merge the efficiency of Dynamic Routing with the simplicity of Robust Routing. We showed that the problem of providing an optimal solution satisfying Volume-Oriented Routing is difficult in general. However, we presented a way to cope with this difficulty, i.e., Simplified Volume-Oriented Routing and General Volume-Oriented Routing, which are polynomially solvable modifications of Volume-Oriented Routing. We showed that Simplified Volume-Oriented Routing cannot outperform General Volume-Oriented Routing as far as cost is concerned. Numerical results proved the applicability of the approaches.
Finally, Volume-Oriented Routing and Simplified VolumeOriented Routing can be easily implemented since only local information is required for routing. Two routing schemes for demand $t_{i j}$ have to be memorized in the routing table of node $i$ : the first scheme is used until the demand volume achieves the threshold $h_{i j}$, and then all extra traffic is routed according to the second scheme. The implementation of General Volume-Oriented Routing is slightly more complex, although still decentralized.

Open Access This article is distributed under the terms of the Creative Commons Attribution Noncommercial License which permits any noncommercial use, distribution, and reproduction in any medium, provided the original author(s) and source are credited.

\section{References}

1. Altın, A., Amaldi, E., Belotti, P., \& Pınar, M. Ç. (2007). Provisioning virtual private networks under traffic uncertainty. Networks, 49(1), 100-115.

2. Ben-Ameur, W. (2007). Between fully dynamic routing and robust stable routing. In Proceedings of DRCN.

3. Ben-Ameur, W., \& Kerivin, H. (2001). Routing of uncertain demands. In Proceedings of INFORMS.

4. Ben-Ameur, W., \& Kerivin, H. (2003). New economical virtual private networks. Communications of the ACM, 46(6), 69-73. 
5. Ben-Ameur, W., \& Kerivin, H. (2005). Routing of uncertain traffic demands. Optimization and Engineering, 6(3), 283-313.

6. Ben-Ameur, W., \& Żotkiewicz, M. (2011). Robust routing and optimal partitioning of a traffic demand polytope. International Transactions in Operational Research, 18(3), 307-333.

7. Ben-tal, A., \& Nemirovski, A. (1999). Robust solutions of uncertain linear programs. Operations Research Letters, 25, 1-13.

8. Bertsimas, D., \& Sim, M. (2003). Robust discrete optimization and network flows. Mathematical Programming, 98, 49-71.

9. Carpenter, T., Heyman, D. P., \& Saniee, I. (1998). Studies of random demands on network costs. Telecommunications Systems, 10(3-4), 409-421.

10. Chekuri, C. (2007). Routing and network design with robustness to changing or uncertain traffic demands. SIGACT News, 38(3), 106-129.

11. Chekuri, C., Shepherd, F. B., Oriolo, G., \& Scutellá, M. G. (2007). Hardness of robust network design. Networks, 50(1), 50-54.

12. Duffield, N. G., Goyal, P., Greenberg, A., Mishra, P., Ramakrishnan, K. K., \& Van Der Merwe, J. E. (1999). A flexible model for resource management in virtual private networks. In Proceedings of ACM SIGCOMM .

13. Feldmann, A., Greenberg, A., Lund, C., Reingold, N., Rexford, J., \& True, F. (2001). Deriving traffic demands for operational ip networks: Methodology and experience. IEEE/ACM Transactions on Networking, 9, 265-279.

14. Fingerhut, J. A., Suri, S., \& Turner, J. S. (1997). Designing leastcost nonblocking broadband networks. Journal of Algorithms, 24(2), 287-309.

15. ILOG (2007). CPLEX 11.0 User's Manual. http://www.lingnan. net/lab/uploadfile/200864184419679.pdf.

16. Karp, R. M. (1972). Reducibility among combinatorial problems. In R. E. Miller \& J. W. Thatcher (Eds.), Complexity of computer computations (pp. 85-103). New York: Plenum.

17. Kouvelis, P., \& Yu, G. (1997). Robust discrete optimization and its applications. Dordrecht: Kluwer Academic.

18. Lisser, A., Ouorou, A., \& Vial, J.-P. Capacity planning under uncertain demand in telecommunications networks (Technical report). France Telecom R\&D, NT/CNET/6570 (1999).

19. Lorenz, D. H., \& Orda, A. (1998). Qos routing in networks with uncertain parameters. IEEE/ACM Transactions on Networking, 6, 768-778.

20. Orlowski, S., Pióro, M., Tomaszewski, A., \& Wessäly, R. (2010). SNDlib 1.0-survivable network design library. Networks, 55(3), 276-286.

21. Ouorou, A. (2006). Robust capacity assignment in telecommunications. Computational Management Science, 3(4), 285-305.
22. Petrou, G., Lemaréchal, C., \& Ouorou, A. (2007). An approach to robust network design in telecommunications. RAIRO. Recherche Opérationnelle, 41(4), 411-426.

23. Sen, S., Doverspike, R. D., \& Cosares, S. (1994). Network planning with random demand. Telecommunications Systems, 3, 1130.

24. Żotkiewicz, M., \& Ben-Ameur, W. (2009). More adaptive robust stable routing. In Proceedings of GLOBECOM.

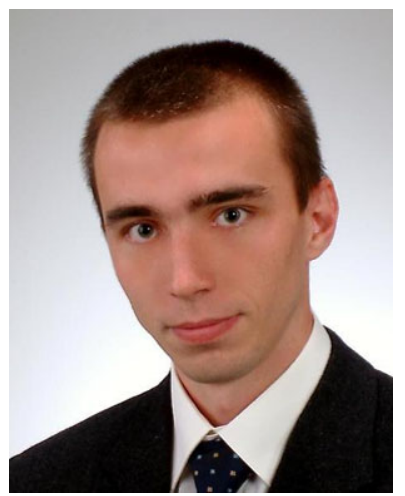

Mateusz Żotkiewicz is an assistant professor at the Computer Networks and Switching Division at the Institute of Telecommunications, Warsaw University of Technology, Poland. He received his Ph.D. degree in telecommunications from Warsaw University of Technology, Poland, and in informatics from Telecom SudParis, France, both in 2011. His research interests concentrate on modeling and design of telecommunications networks.

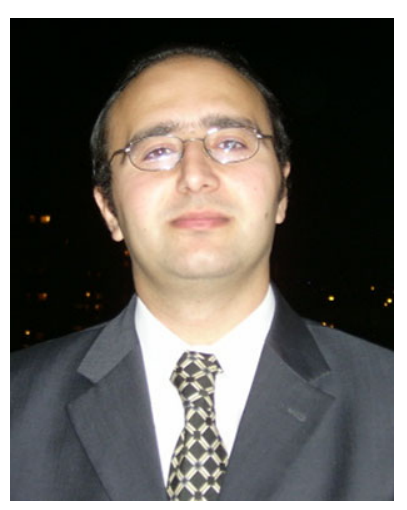

Walid Ben-Ameur obtained B.Sc., M.Sc. and a Ph.D. degrees from the Ecole Nationale Superieure des Telecommunications, Paris, France (Telecom ParisTech). He received a "research habilitation" in Operation Research from University Pierre et Marie Curie. He was with Orange Labs from 1999 to 2001. $\mathrm{He}$ is now a full professor in Telecom SudParis. His main research interests are combinatorial optimization, mathematical programming and network optimization. Professor Ben-Ameur was a winner in 2006 of the Robert Faure prize (a national french prize of operation research) and a co-winner of the Glover-Klingman prize in 2007. 